\title{
Complicated Gastric Ulcer and Jejunal Hematoma with Concomitant Warfarin Use: Analytical and Biomedical Case Study Report
}

\author{
Ali A. Samaha ${ }^{1,2}$, Hilana Hatoum ${ }^{3,4}$, Shaimaa Matar ${ }^{4}$, Fouad Zaarour ${ }^{4}$, John J. Haddad ${ }^{5, *}$ \\ ${ }^{1}$ Faculty of Public Health, Lebanese University, Zahle, Lebanon \\ ${ }^{2}$ Department of Biomedical Sciences, Lebanese International University, Beirut, Lebanon \\ ${ }^{3}$ Department of Gastroenterology, Sahel General Hospital, Beirut, Lebanon \\ ${ }^{4}$ Faculty of Medicine, Lebanese University, Beirut, Lebanon \\ ${ }^{5}$ Cellular and Molecular Physiology and Immunology Signaling Research Group, Biomedical Laboratory and Clinical Sciences \\ Division, Department of Medical Laboratory Technology, Faculty of Health Sciences, Beirut Arab University, Beirut, Lebanon \\ *Corresponding author: john.haddad@yahoo.co.uk
}

Received May 27, 2013; Revised June 19, 2013; Accepted June 20, 2013

\begin{abstract}
Intramural hematoma is commonly known as a hematoma that occurs in the wall of a structure, such as the bowel or bladder, usually resulting from trauma or, in rare situations, excessive anticoagulation. In particular, intramural gastrointestinal hematoma is part of a spectrum of gastrointestinal injuries. Vomiting and straining, endoscopic procedures, and bleeding disorders are essentially among the most common predisposing factors. However, it can also be an unusual complication of anticoagulation and/or thrombolysis therapy. The most common symptoms are retrosternal chest pain, dysphagia, and hematemesis. Intramural bowel wall hematoma is an often forgotten, rare clinical entity that may pose considerable diagnostic dilemma. Therefore, it is conspicuously imperative to diagnose this condition promptly and correctly in order to avoid unnecessary surgical intervention. In this rare presented case report, a 51-year-old woman having mitral valve prothesis and mild heart failure under warfarin wasadmitted to the renowned Sahel General Hospital in Beirut in November of 2006 with massive upper gastrointestinal bleeding that was subsequently controlled by endoscopic sclerotherapy and medical management. About $12 \mathrm{~h}$ later, the aforementioned patientsuffered a severe abdominal pain accompanied by a steep dropping in the hematocrit level. Moreover, an abdominal computed tomography scan showed thick jejunal hematoma of approximately $70 \mathrm{~cm}$ in length that was handled surgically. The patient had been incessantly observed for about 7 days,andsubsequently discharged with therapeutic international normalized ratio(INR).
\end{abstract}

Keywords: CT, Hemorrhagic bowel syndrome, INR, jejunal hematoma, NSAIDs, warfarin

\section{Introduction and Background}

In the past several years, there have been an increasing number of reports, albeit rare and uncommon, of jejunal hemorrhage syndrome (JHS) [1,2,3]. This condition, also known as hemorrhagic bowelsyndrome (HBS), bloody gut syndrome, dead gut, and enterotoxaemia, affects the small intestine, specifically at the level of the jejunum. The first preliminary reports of JHS in the scanned literature come from the early nineties in northeastern US, but since that time there have been confirmed cases reported throughout the US and the world [3,4,5]. The syndrome technically appears without evidential warning signs, and is frequently fatal. Furthermore, periods of concentrated problems are often interspersed by intervals free of any apparent disease, thereby complicating the situation. Although the JHS/HBS syndrome targets the jejunum of affected individuals, the most severely inflicted have more extensive involvement of the small intestine [4,5].
Intramural hematoma, especially jejunal hematoma (JHS),is a very rare disease. JHSespecially affects the pediatric population more often than the adult population, whilst it occurs in both genders with a slight, but significant, male predominance in children [4,5,6,7]. It is well known that the duodenum is the most common site for JHS, with the first reported medical case appearing back in 1938, when it was thought to be a "pseudoaneurysm between the mucosa and muscular layers."Furthermore, statistically more than $70 \%$ of cases are usually associated with trauma $[6,7,8]$. Interestingly, JHS is usually ruled out in child abuse cases if the problem had occurred prior to 5 years of age (Figure 1 and Figure 2).

\section{Case Report Presentation}

In our case, a 51-year-old woman, a smoker, had a metallic mitral valve installed since 2001 due to rheumatic heart disease with mild heart failure NYHA II under warfarin $4 \mathrm{mg}$ ( $3 / 4$ tablet daily), furosemide $40 \mathrm{mg}$, 
captopril $25 \mathrm{mg}$, amiodarone 200mg, and simvastatin 10 mg. Moreover, she had taken diclofenac $100 \mathrm{mg}$ daily for back pain for one week, ostensibly without the knowledge of her physician. Following that, she was presented to the emergency room with acute onset of hematemesis (fresh blood seven times), associated with continuous crampy localized epigastric pain starting few minutes before hematemesis, albeit without vomiting, anorexia, melena, rectorrhagia, weight loss, fever, chills or other respiratory or cardiac symptoms. In the ER, she was with tachycardia ( $\mathrm{P}=100$ beats $/ \mathrm{min}$.), tachypnea( $\mathrm{RR}=24 \mathrm{beats} / \mathrm{min}$.), afebrile $\left(37^{\circ} \mathrm{C}\right)$ with conserved BP $(130 / 70 \mathrm{mmHg})$. Moreover, she was pale, diaphoretic, and with dyspnea but conscious and well oriented. On auscultation, she had regular heartbeats, mitral click, and diffuse inspiratory and expiratory wheezes; her abdomen was essentially normal except that with mild epigastric pain on palpation, no hepatosplenomegaly, and no hepatojugular reflux $[5,6,7,8,9,10]$. Moreover, she had few ecchymosis on her four limbs with positive peripheral pulses.

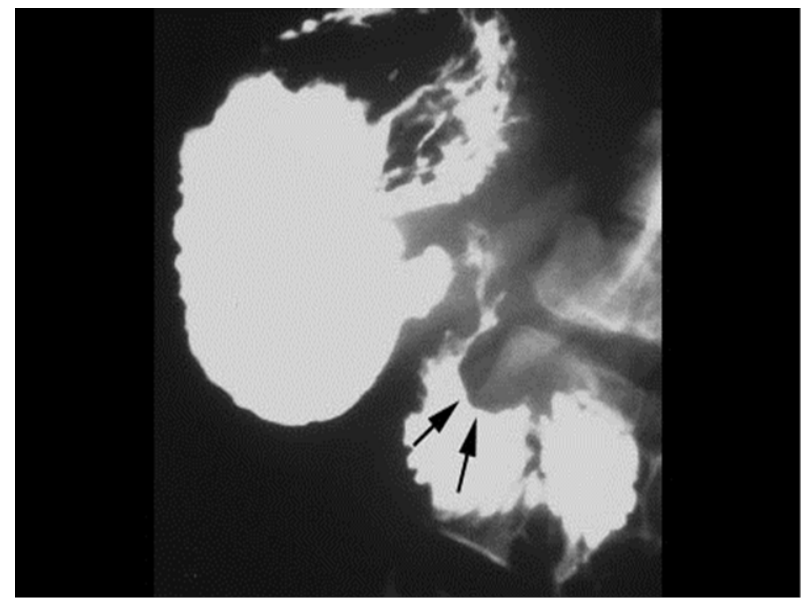

Figure 1. Barium meal of duodenal hematoma

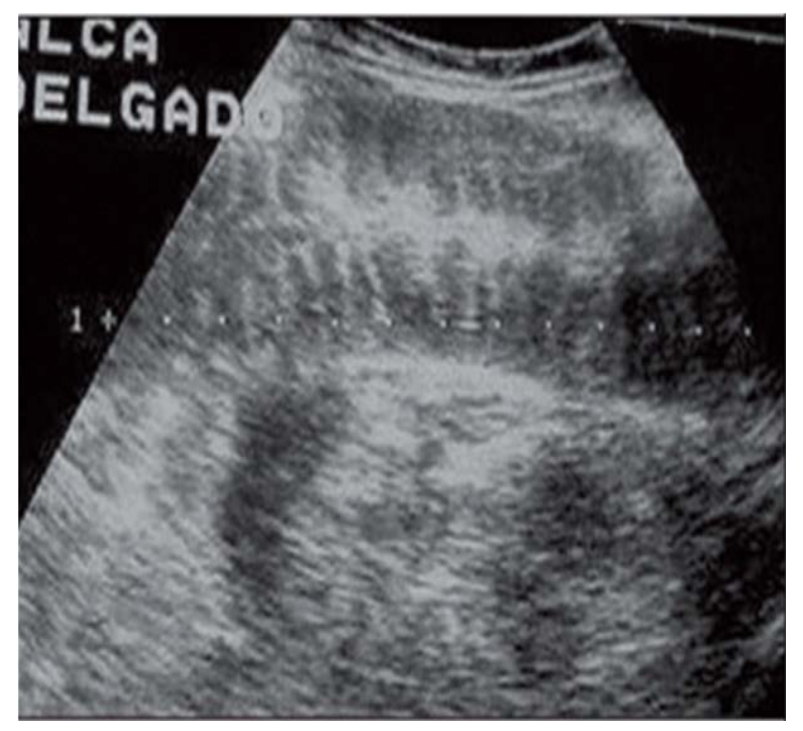

Figure 2. Ultrasound of duodenal hematoma

In further evaluating her complicated condition, an EGD urgently performed revealed the presence of cardiac ulcer with a clot and severe antral gastritis (Figure 3, Figure 4, and Figure 5); a hemostasis with adrenaline injection was performed and the patient was subsequently transferred to the ICU for close observation. Furthermore, laboratory tests were normal except that for prolonged PTT (120sec), international normalized ratio (INR; >9), high BUN and LDH, normal creatinine and hematocrit of 33 (Table 1).

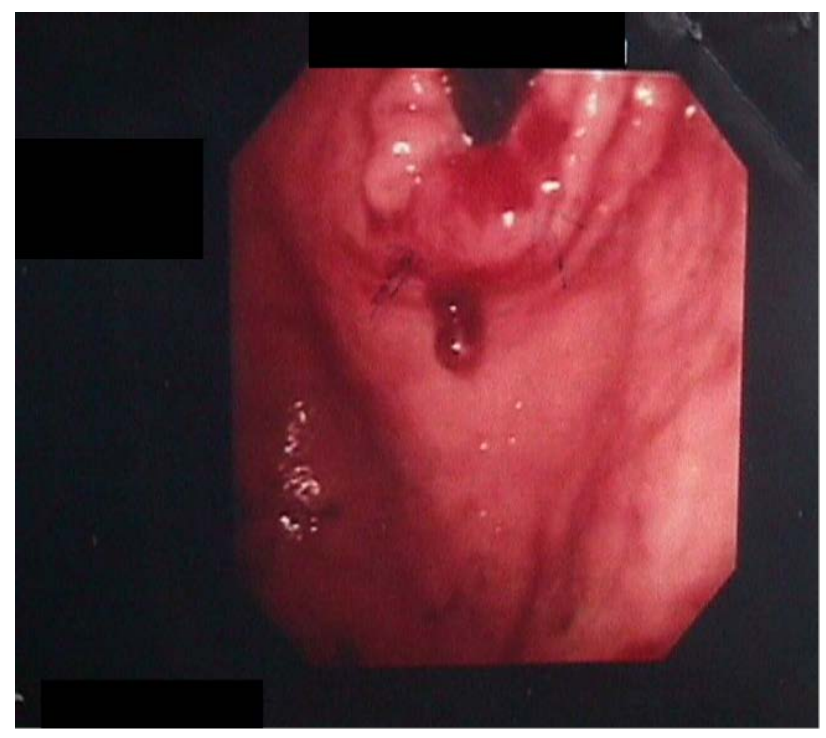

Figure 3. Gastroscopy of the case showing cardiac ulcer with a blood clot

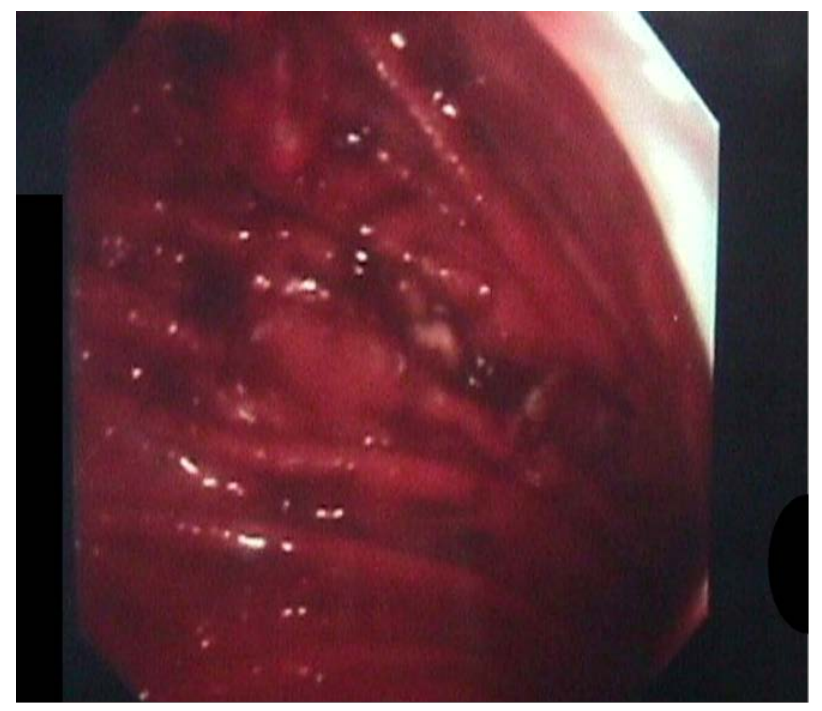

Figure 4. Gastroscopy of the case showing intragastric bleeding

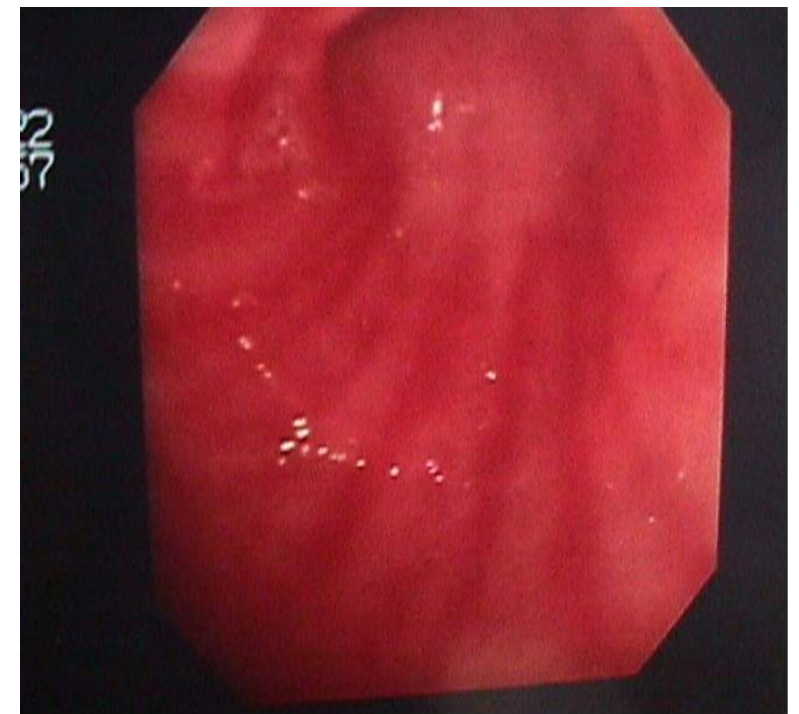

Figure 5. Gastroscopy of the case showing antral gastritis 
Table 1. Patient's laboratory results

\begin{tabular}{|c|c|}
\hline \multicolumn{2}{|c|}{ Laboratory Results on Admission } \\
\hline Hemoglobin & 11 \\
\hline Hematocrit & 33 \\
\hline Platelets & 200,000 \\
\hline BUN & 62 \\
\hline Creatinine & 0.9 \\
\hline INR & $>9$ \\
\hline PTT & 120 \\
\hline MCV & 88.6 \\
\hline LDH & 295 \\
\hline Na & 140 \\
\hline K & 4.5 \\
\hline Cl & 105 \\
\hline HCO3 & 25 \\
\hline SGPT & 50 \\
\hline SGOT & 45 \\
\hline GGT & 30 \\
\hline Phosp Alk & 35 \\
\hline Amylase & 38 \\
\hline Lipase & 33 \\
\hline
\end{tabular}

About $12 \mathrm{~h}$ later, the patient started to complain of diffuse severe abdominal pain with observable decrease in the hematocrit level from 33 to 27, but no more overt bleedinghad occurred. Two units of PRCR were transfused and an urgent abdominal computed tomography (CT) scan showed the presence of a thick mural jejunum with free intra-abdominal fluid going with blood and intramural hematoma (Figure 6, Figure 7, and Figure 8). The patient subsequently underwent surgery with a jejunal resection of $70 \mathrm{~cm}$ and drainage of intra-abdominal blood.Medical management was undertaken with vitamin $\mathrm{K}$ once, four fresh frozen plasma, blood transfusion of another two units of PRBC, IV fluids, and close observation of the urine output and vital signs $[8,9,10,11,12,13]$.

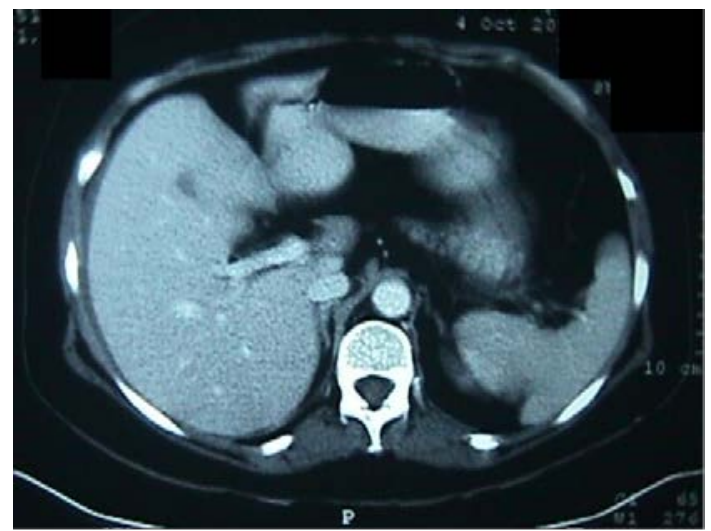

Figure 6. CT scan showing a thick jejunum of $15 \mathrm{~mm}$.

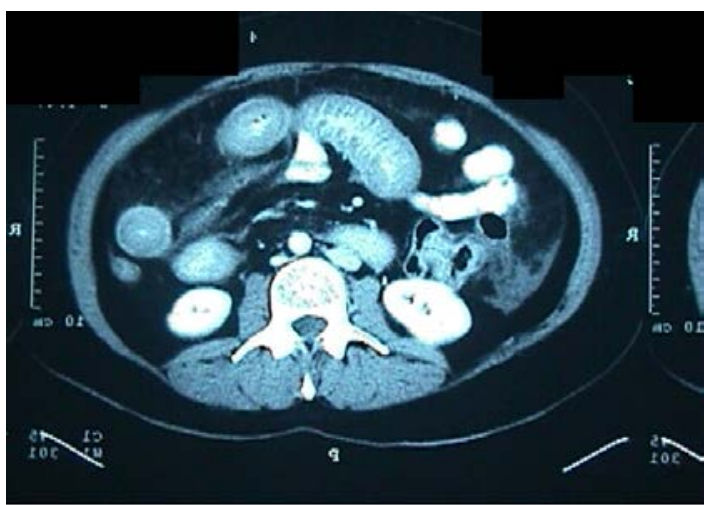

Figure 7. CT scan showing a thick jejunum

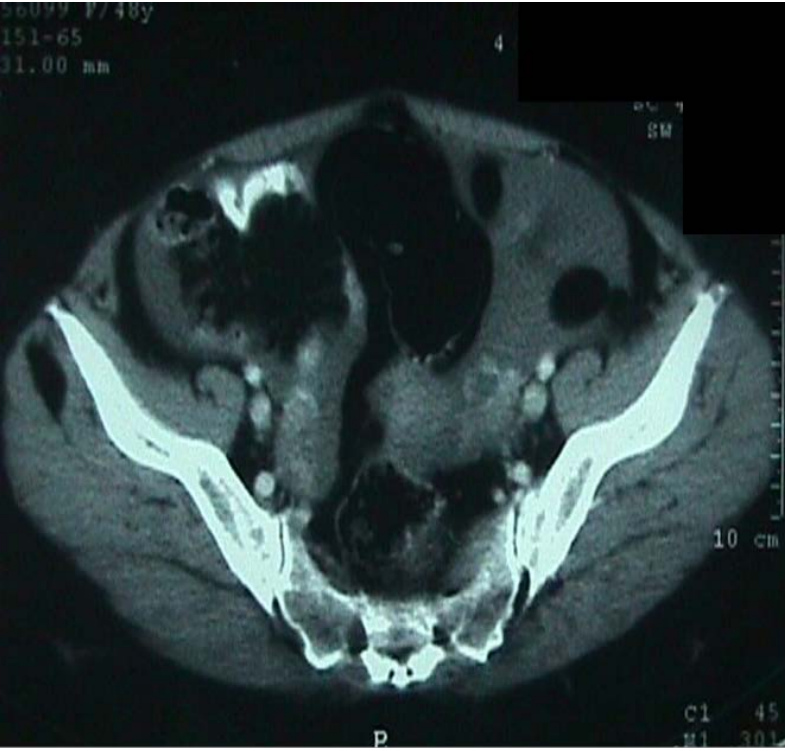

Figure 8. CT scan showing an intra-pelvic fluid collection

Moreover, the patient's INR decreased gradually from 9 $\rightarrow 5 \rightarrow 3 \rightarrow 2$, then 1.3; at the INR value of 2 heparin was administered with close watching of PTT. On day five post-surgery, she was clinically stable, having bowel movement, and fluid intake was started gradually, and she was transferred to the regular floor for another 3 days, while enteral feeding has been activated and sutures removed. Ambulation was commenced and warfarin resumed with low dose to be discharged under $2 \mathrm{mg}$ warfarin daily with an INR of 2.5. Another outpatient follow-up was performed almost two weeks after her discharge. The patient was stable, with no tangible complaints, and her physical exam was essentially normal, her INR was 2.5, and hematocrit 34. A monthly follow-up was performed with no apparent changes $[12,13,14,15]$.

\section{Discussion}

Intramural jejunal hematoma is characterized by a hemorrhagic episode that starts within the submucosa of the jejunum. The collection of blood forms a hematoma and may eventually progress to dissection of the submucosal layer $[16,17,18]$. JHS is part of a spectrum of gastrointestinal injuries that range from local mucosal tears to transmural rupture of the jejunum. The several subtypes of JHS are categorized by the nature of the hemorrhage: i) Traumatic; ii) Emetogenic; iii) Abnormal hemostasis-related; iv) Aorta-related; and v) Spontaneous [19-25]. In particular, spontaneous submucosal hematoma of the jejunum is further sub-classified into factors that increase the tendency of bleeding such as drugs or an underlying acute or chronic disease [1,3,10,12,22,26-30].

Although risk factors vary, the most common predisposing factors for JHS are endoscopic procedures, vomiting and straining, and bleeding disorders. JHS is more commonly seen in middle-aged patients, with a slight predominance in females. The clinical stages of JHS manifestation are evaluated according to the degree of luminal involvement [25-30]. Stage I and Stage II are characterized by isolated hematoma and hematoma with surrounding tissue edema, respectively. Stage III involves a hematoma with edema and compression of the lumen, 
while Stage IV is the complete obliteration of the jejunal lumen with formation of hematoma, edema, and organized clot formation (Table 2) [31,32,33,34,35].

Table 2. Causes of bowel hematoma.

\begin{tabular}{|l|l|}
\hline $\begin{array}{l}\text { Causes of bowel } \\
\text { hematomas }\end{array}$ & Examples: \\
\hline Platelets dysfunction & $\begin{array}{l}\text { Glansman's thrombasthenia } \\
\text { Bernard-Soulier Sd }\end{array}$ \\
\hline Platelets deficiency & $\begin{array}{l}\text { ITP / TTP } \\
\text { HUS / Leukemia } \\
\text { Severe hypersplenism }\end{array}$ \\
\hline Drugs & Heparin / warfarin / thrombolytic \\
\hline Trauma & $\begin{array}{l}\text { Abdominal / endoscopic manipulation/ } \\
\text { forceful vomiting }\end{array}$ \\
\hline Vasculitis & $\begin{array}{l}\text { Polyarthritis nodosa / henoch schonlein } \\
\text { purpura / Ehlers-Danlos Sd }\end{array}$ \\
\hline
\end{tabular}

Speaking of which, warfarin is extensively used for therapeutic and prophylactic purposes [1-5]. The most important complication of the anticoagulation treatment with warfarin is bleeding. It is associated with various hemorrhagic complications, including hematuria, gastrointestinal bleeding, intracerebral hemorrhage, soft tissue hematoma, epistaxis, and retroperitoneal hematoma. Bleeding, which is presented as an intramural hematoma of the small intestine, is a rare complication that is seen in 1 out of approximately 2,500 patients. Excessive anticoagulation with warfarin is the most common cause of spontaneous, intramural small bowel hematoma (JHS). Other risk factors may include hemophilia, idiopathic thrombocytopenic purpura, leukemia, lymphoma, myeloma, chemotherapy, vasculitis, pancreatitis, and pancreatic cancer.The presentation can vary from a mild abdominal pain to intestinal obstruction, and an acute abdomen. If suspected pre-operatively, the diagnosis usually requires CT scan for its confirmation. Most of the patients can be treated non-operatively, ostensibly with a good outcome [12,13,14,15,25-32].

Anticoagulation and/or thrombolysis therapy is an unusual cause of JHS, with only a handful of documented cases, especially in the absence of endoscopic trauma or bleeding disorders. Chest pain, dysphagia/odynophagia, and hematemesis are the most common initial symptoms of JHS [30-35]. There are approximately 35\% of patients who present with this triad of symptoms, while at least $50 \%$ of patients present with at least two of the above symptoms. As such, it is important to differentiate JHS from several ischemic events because although it is a relatively benign condition, it could be worsened by anticoagulation therapy. In the setting where anticoagulation and/or thrombolysis are necessary, such as a PE, it may be beneficial to perform periodic reassessments for new-onset chest pain and dysphagia/odynophagia so JHS can be recognized and managed earlier [36,37,38].

As we have indicated earlier on, intramural bowel hematomas are rare and the JHScondition in particular is usually a very rare entity. The causes of bowel hematoma are essentially classified as follows: i) Platelets dysfunction and deficiency; ii) Drugs; iii) Trauma; and iv) Vasculitis (see Table 1).The diagnosis is practically based on clinical (symptoms and signs of abdominal obstruction due to mass effect or ileus post bleeding) and radiological findings [38-42].Furthermore, enhanced CT is considered one of the best diagnostic methods. In addition, ultrasound and barium meal are also useful (see Figure 1). On the other hand, endoscopic studies are usually performed in inconclusive cases [1,5,8,12,16,25,28,33,37].

Interestingly, the treatment is usually surgical therapy (first line after 1970s) as radial incision of the bowel for evacuation or excision with or without bypass surgery. Conservative therapy was used as the first line therapy before the 1970s, mainly in small isolated cases via putting the patient with rest bowel, nasogastric (NG) suction, and central hyper alimentation, or via CT-guided percutaneous aspiration with somatostatin use in severe panceatico-duodenal damage or periodic closing NG (usually to test and stimulate duodenal transit) [35-45]. Moreover, it is well established that complications are secondary to surgical treatment, including: i) Pancreatitis (traumatic/post-operatory); ii) Iatrogenic mucosal leakage; iii) Intestinal ileus; and iv) Malabsorption of resection of long bowel. More importantly, prognosis is usually good and this depends on the associated comorbidities and the site or length of the affected bowel [17,18,19,22-27].

In returning to our case, the patient was under warfarin and had taken non-steroidal anti-inflammatory drugs (NSAIDs)as she came with upper gastrointestinal bleeding. The cause was determined by the endoscope findings as "NSAIDs-induced gastric ulcer and severe gastritis." At that time, the patient was treated by endoscopic sclerotherapy with medical management, in addition to the correction of her prolonged INR. Few hours later, she complained of abdominal severe pain with a drop in her hematocrit level $[1,20]$. The possible diagnostic observations at that time were including the following: i) Rebleeding from the same ulcerogenic site; ii) Irritation of the gastric mucosa by nasogastric tube; iii) Bleeding from another site; iv) Perforation of the ulcer; and v) Vague abdominal pain due to patient's problem with dilution of hematocrit [28-36].

On physical examination, she was hemodynamically stable, as there was no bleeding from NG tube, and abdomen was soft, with no rebound tenderness with diffuse abdominal pain on palpation. Furthermore, no hematuria or other sites of bleeding were observed. Laboratory tests revealed that the hematocrit decreased from 33 to 27. In addition, an abdomino-pelvic CT scan eliminated the presence of free intra-abdominal air, therefore, the possibility of perforation was eliminated, but it showed a thickness of approximately $15 \mathrm{~mm}$ in the jejunum with free fluid intra-abdominally going with blood hematoma. The diagnosis of $\mathrm{JH}$ as another site of bleedingprobably explained the drop in hematocrit and the patient's recurrent abdominal pain [10,11,12,20,21,42-45].

Promptly, the surgeons decided to undergo the surgery to prevent bleeding and avoid intestinal necrosis. A jejunectomy was performed on the following day with resection of $70 \mathrm{~cm}$ from the small bowel, in addition to the suction of free blood in the abdomen. The pathologic examination of the resected part confirmed an intramural bleeding with no presence of abnormal cells, any underlying vasculitis, or other malignant processes [1-5].

Finally, the routine management of gastrointestinal bleeding was performed (hydration, blood transfusion, 
bowel rest, correction of INR, and urine output). During her observation in the ICU, the patient was stable hemodynamically almost all the time, with no more pain, NG bleeding, and per rectum or hematuria. Moreover, the physical exam was normal with mild wheezes due to her underlying chronic obstructive pulmonary disease (COPD).She passed gas on the third post-surgery, and her hematocrit level was stable. An enteral feeding was started gradually with good tolerance, and she was subsequently transferred to a regular floor following 5 days at ICU. On the floor, she started moving with reintroduction of warfarin in order to put her INR in the therapeutic range. Few days after, her INR was two and she was discharged home one week later with a therapeutic dose of warfarin. Several follow-upsdone at two, four weeks, and 3 months following discharge demonstrated normal physical exam, no more complaints, and therapeutic INR [1,20-23].

\section{Conclusions and Remarks}

Intramural hematoma of the jejunum is a rare complication of anticoagulation therapy. In 1965, Walter Goldfarb published a series of eleven patients with intestinal hemorrhage that was related to oral anticoagulation therapy $[1,2,3,4,5]$. Warfarin toxicity remains the dominant cause, accounting for the vast majority of patients. The small bowel is affected in up to $85 \%$ of the occurrences of haematoma, with the jejunum being the most affected region, in contrast to the posttraumatic findings that affect the duodenum more. The incidence of spontaneous intramural haematoma is reported to be 1 per 2,500 anti-coagulated patients. The mean age at presentation in one recent series of 13 patients was 64 years; $15 \%$ of the patients in this series had multiple hematoma $[1,2,3]$.

The presentation can vary from mild, vague abdominal pain to intestinal obstruction and an acute abdomen. The hemorrhage is usually located in the submucosal layer of the bowel and it originates from a small vessel that produces slow bleeding. Hemorrhagic ascites can be present and it is related to the leakage of blood from an engorged, thickened and inflamed bowel wall, with the submucosal bleeding extending into all the layers. In addition to intramural bleeding, intraluminal, intramesenteric and retroperitoneal hemorrhage can also occur, especially when the duodenum is involved. Abdominal CT is the key for its diagnosis, with the characteristics including circumferential wall thickening, intramural hyperdensity, luminal narrowing, intestinal obstruction, and hyperdense ascites $[15,16,17,18]$. The first step in the treatment of acute intramural small bowel hematoma is the discontinuation of the anticoagulant medication and the correction of the coagulation parameters with fresh-frozen plasma and vitamin $\mathrm{K}$. Operative intervention is only indicated if there is significant intraluminal hemorrhage, bowel perforation or ischemia [18,19,20,21,22].

As noted, small bowel hematoma is a rare clinical entity. It should be considered in any patient on long-term anticoagulation therapy, who present with an acute abdomen. CT, especially non-enhanced CT, is a valuable tool in the diagnosis of this condition. An early diagnosis is crucial, because most of the patients can be treated nonoperatively, with a good outcome.

\section{Authors' Contributions}

All authors have squarely and equally contributed to developing the experimental, theoretical and statistical aspects of this article.

\section{Declaration of Competing Interests}

The authors declare that they have no competing interests.

\section{Acknowledgments}

The authors would like to thank their colleagues at Sahel General Hospital for clinical support and critical assessment.

\section{References}

[1] Kumar KP, Diaz EAI, Mascarenhas RM.A rare cause of acute abdomen due to spontaneous intramural haematoma of the jejunum in a patient on warfarin. J Clin Diag Res 2011; 5: 1291-2.

[2] Jaskolka JD, Binkhamis S, Prabhudesai V, et al. Acute gastrointestinal hemorrhage: Radiologic diagnosis and management. Can Assoc Radiol J 2013; 64: 90-100.

[3] Avent ML, Canaday BR, Sawyer WT. Warfarin-induced intramural hematoma of the small intestine. Clin Pharm 1992; 11: 632-5.

[4] Kleinman PK, Brill PW, Winchester P. Resolving duodenaljejunal hematoma in abused children. Radiol 1986; 160: 747-50.

[5] Moody EE. Acute jejunal obstruction secondary to traumatic intramural hematoma. Pediatrics 1957; 19: 863-8.

[6] Birla RP, Mahawar KK, Saw EYW, et al. Spontaneous intramural jejunal haematoma: A case report.Cases J 2008; 1: 389.

[7] Oyasiji T, Yood S.Jejunal amyloidoma: A rare cause of gastrointestinal bleeding.Cases J 2009; 2: 9100.

[8] Essenhigh DM, Chir M, Toland J. Duodeno-jejunal haematoma. Br J Radiol 1968; 41: 349-53.

[9] Hou SW, Chen CC, Chen KC. Sonographic diagnosis of spontaneous intramural small bowel haematoma in a case of warfarin overdose. J Clin Ultrasound 2008; 36: 374-6.

[10] Uzun MA, Koksal N, Gunerhan Y. Intestinal obstruction due to spontaneous intramural haematoma of the small intestine during warfarin use: A report of two cases. Eur J Emerg Med 2007; 14: 272-3.

[11] Abbas MA, Collins JM, Olden KW. Spontaneous intramural small-bowel haematoma: imaging findings and outcome. AJR Am J Roentgenol 2002; 179: 1389-94.

[12] Tran F, Koo JH. Gastrointestinal: Spontaneous jejunal hematoma secondary to supratherapeutic anticoagulation.J Gastroenterol Hepatol 2012; 27: 1643.

[13] Abrassart S, Stern R, Peter R.Unstable pelvic ring injury with hemodynamic instability: What seems the best procedure choice and sequence in the initial management? Orthop Traumatol Surg Res 2013; 99: 175-82.

[14] Spinelli G, Damiano G, Palumbo VD, et al. Ileus following spontaneous jejunum intramural hematoma: Case report and review of the literature. Acta Clin Croat 2012; 51: 435-9.

[15] Erkan G, Çalişkan A, Kaan Ataç G, et al. Spontaneous intramural jejuna hematoma: Two cases. Turk J Gastroenterol 2012; 23: 6156.

[16] Abdel Samie A, Sun R, Huber A, et al. Spontaneous intramural small-bowel hematoma secondary to anticoagulant therapy: A case series. Med Klin Intensivmed Notfmed 2013; 108: 144-8.

[17] Abdel Samie A, Theilmann L.Detection and management of spontaneous intramural small bowel hematoma secondary to anticoagulant therapy. Expert Rev Gastroenterol Hepatol 2012; 6: 553-8.

[18] Chen H, Bai YZ, Wang WL.Jejunal subserosal hematoma in an 11-year-old boy. J Gastrointest Surg 2011; 15: 2291-2. 
[19] Chong VH, Basir N, Yaakub AB.Acute pancreatitis complicated by jejuna hematoma in a patient on anti-coagulants and antiplatelets. JOP 2010; 11: 262-5.

[20] Cheng J, Vemula N, Gendler S.Small bowel obstruction caused by intramural hemorrhage secondary to anticoagulant therapy. Acta Gastroenterol Belg 2008; 71: 342-4.

[21] Chaiteerakij R, Treeprasertsuk S, Mahachai V, et al. Anticoagulant-induced intramural intestinal hematoma: report of three cases and literature review. J Med Assoc Thai 2008; 91: 1285-90.

[22] Chen YW, Chen MH, Wang LM, et al. Spontaneous intramural intestinal haematoma. Intern Med J 2007; 37: 661-2.

[23] Shinozaki S, Yamamoto H, Kita H,et al. Direct observation with double-balloon enteroscopy of an intestinal intramural hematoma resulting in anticoagulant ileus. Dig Dis Sci 2004; 49: 902-5.

[24] Lobo L, Koudki R, Prasad HIK, et al.Colon obstruction due to an anticoagulant induced intramural haematoma; A Rare Case Report. J Clin Diagn Res 2013; 7: 739-41.

[25] Cao DT, Reny JL, Lanthier N, et al.Intramural hematoma of the esophagus. Case Rep Gastroenterol 2012; 6: 510-7.

[26] Hong M, Warum D, Karamanian A. Spontaneous intramural esophageal hematoma (IEH) secondary to anticoagulation and/or thrombolysis therapy in the setting of a pulmonary embolism: A case report. J Radiol Case Rep 2013; 7:1-10.

[27] Pomara C, Bello S, D’Errico S, et al.Sudden death due to a dissecting intramural hematoma of the esophagus (DIHE) in a woman with severe neurofibromatosis-related scoliosis. Forensic Sci Int 2013; 228:e71-5.

[28] Chen HL, Wu CC, Lin AC. Small bowel intramural hematoma secondary to abdominal massage. Am J Emerg Med 2013; 31:758.e3-4.

[29] Silva JD, Veloso N, Godinho R, et al. Fatal acute pancreatitis following sclerosis of a bleeding duodenal ulcer complicated by an intramural duodenal hematoma. Rev Esp Enferm Dig 2012; 104:603-4.

[30] Sun P, Tan SY, Liao GH. Gastric intramural hematoma accompanied by severe epigastric pain and hematemesis after endoscopic mucosal resection. World J Gastroenterol 2012; 18:7127-30.

[31] McClain LE, Pullatt RC. Spontaneous intramural hematoma of the ileum associated with obstruction during low-molecular-weight heparin use. Am Surg 2013;79:E3-5.
[32] Jeong ES, Kim MJ, Yoo SH, et al. Intramural hematoma of the esophagus after endoscopic pinch biopsy. Clin Endosc 2012; 45:417-20.

[33] Ikegami R, Tsuchida K, Oda H.Acute myocardial infarction caused by spontaneous coronary intramural hematoma. $J$ Invasive Cardiol 2012;24:692-3.

[34] Chen CY, Yan YH.Duodenal dissection secondary to intramural haematoma after endoscopic therapy for a bleeding duodenal ulcer in a patient on long-term haemodialysis with liver cirrhosis. BMJ Case Rep 2012;2012.

[35] Julià I, Tauron M, Muñoz-Guijosa C. Postpartum acute coronary syndrome due to intramural hematoma and coronary artery dissection. Thorac Cardiovasc Surg 2013; 61:85-7.

[36] Droste J, Zadeh HZ, Arif M, et al. Acute aortic intramural hematoma presenting with painless recurrent syncope. Heart Surg Forum 2012; 15:E286-8.

[37] Goyal H, Singla U, Agrawal RR. A rare cause of acute pancreatitis: Intramural duodenal hematoma. Case Rep Gastrointest Med 2012; 2012:275604.

[38] Johnson TW, Smith D, Strange JW, et al.Spontaneous multivessel coronary intramural hematoma: An insight with OCT. JACC Cardiovasc Imaging 2012;5:1070-1.

[39] Iyisoy A, Celik M, Celik T, et al.The role of intravascular ultrasound guidance in the treatment of intramural hematoma probably caused by spontaneous coronary artery dissection in a young woman with acute anterior myocardial infarction. Cardiol $J$ 2012; 19:532-5.

[40] Harris KM, Braverman AC, Eagle KA, et al.Acute aortic intramural hematoma: An analysis from the International Registry of Acute Aortic Dissection.Circulation 2012;126:S91-6.

[41] Lee JY, Chung JS, Kim TH.Successful endoscopic decompression for intramural duodenal hematoma with gastric outlet obstruction complicating acute pancreatitis.Clin Endosc 2012;45:202-4.

[42] Costelloe J, McCormack O, Reynolds JV. Dissecting intramural hematoma of the esophagus. Dis Esophagus 2013;26:346.

[43] Sarici IS, Ozçinar B, Bekın A.Intramural small bowel hematoma secondary to use of oral anticoagulant therapy. Turk $J$ Gastroenterol 2012;23:88-91.

[44] Barbetakis N, Asteriou Ch, Kleontas A, et al. Spontaneous intramural esophageal hematoma. Hippokratia 2011;15:280.

[45] Shankarnaryanan S, Hardikar W.Unusual cause of neonatal rectal bleeding: Colonic intramural haematoma. J Paediatr Child Health 2012; 48:E108-9. 